\title{
МЕТОДОЛОГИЯ ПРОГНОЗИРОВАНИЯ ТЕХНИКО-ЭКОНОМИЧЕСКИХ ПАРАМЕТРОВ ПРЕДПРИЯТИЯ В УСЛОВИЯХ НЕОПРЕДЕЛЕННОСТИ
}

\author{
(C) 2020 Суменков Михаил Сергеевич \\ доктор экономических наук, профессор \\ Уральский государственный юридический университет, Россия, Екатеринбург \\ E-mail: ssm0001@yandex.ru \\ (c) 2020 Суменков Сергей Михайлович \\ кандидат экономических наук, доцент, кафедра предпринимательского права \\ Уральский государственный юридический университет, Россия, Екатеринбург \\ E-mail:ssm0001@yandex.ru \\ (c) 2020 Новикова Наталья Юрьевна \\ старший преподаватель кафедры финансовых рынков и банковского дела \\ Уральский государственный экономический университет, Россия, Екатеринбург \\ E-mail: novikova_ny@mail.ru
}

В статье рассмотрены методологические основы производства сложного реального прогноза, когда приходится иметь дело с совокупностью детерминированных, стохастических, неопределенных факторов. При этом наибольшую сложность представляет учет неопределенных, а также стохастических факторов.

Ключевые слова: прогнозирование, детерминированные факторы, стохастические факторы, неопределенные факторы, прогнозирующая система, корреляционный анализ, регрессионный анализ, математический анализ.

При производстве прогноза необходимо принимать во внимание ряд факторов, влияющих на исследуемый процесс. Данные факторы можно разбить на управляемые и неуправляемые. Управляемыми считаются те факторы, посредством изменения которых мы добиваемся конечной цели. Их совокупность определяет возможности управления экономической системой. К неуправляемым относятся факторы, влияние которых на процесс не зависит от строения системы. Это:

- детерминированные факторы - фиксированные неслучайные величины, значения которых известны;

- стохастические факторы - случайные величины с известными законами распределения;

- неопределенные факторы - факторы, относительно которых ни их значения, ни закон распределения не известны.

Обычно при производстве сложного реального прогноза приходится иметь дело с совокупностью факторов всех перечисленных групп, когда задача прогнозирующей системы заключается в установлении прогнозируемых параметров экономической системы с учетом влияния на процесс всех неуправляемых факторов. При этом наибольшую сложность представляет учет неопределенных, а также стохастических факторов.

Точный учет неопределенности сопряжен со значительными экономическими затратами и в ряде случаев невозможен, т.е. у прогнозиста всегда есть вероятность ошибиться в своем прогнозе, и хотя прогноз в условиях неопределенности менее точен, чем прогноз в детерминированном случае, в последнее время мы имеем дело, как правило, с экономическими системами, функционирующими в условиях неопределенности, поэтому разработка методических положений для осуществления прогноза для таких систем необходима.

В зависимости от факторов, влияющих на ход прогнозируемого процесса, все прогнозы можно разбить на три больших класса:

1. Прогноз в условиях определенности. Этот наиболее изученный вид прогнозов характеризуется тем, что на ход процесса оказывают влияние лишь неуправляемые детерминированные факторы. В данном случае существует однозначная детерминированная связь между дина- 
мическим рядом прогнозируемого параметра и его прогнозом. Поскольку детерминированные факторы полностью известны и их действие неизменно приводит к конкретному результату, прогноз в условиях определенности обычно не вызывает теоретических затруднений и связан лишь с трудностями вычислительного характера.

2. Прогноз в условиях неполной определенности (в условиях риска). К этому классу относятся прогнозы, которые производятся в условиях влияния стохастических факторов, т.е. случайных величин, значения которых неизвестны, но известен закон их распределения. В этом случае для каждого конкретного значения стохастического фактора можно определить прогноз параметра и вероятность его осуществления. Знание статистических характеристик стохастических параметров (закона распределения, математического ожидания, дисперсии) позволяет произвести прогноз на основании усредненных значений последних. Однако несмотря на то, что полностью известна информация о ходе процесса, известны все его статистические характеристики, в каждом конкретном случае значение прогнозируемого параметра заранее неизвестно. В этом смысле всегда существует риск получить результат, отличающийся от прогноза, выработанного на основании усредненных статистических характеристик стохастических параметров.

3. Прогноз в условиях неопределенности. Этот наиболее сложный вид прогнозов производится в условиях действия неопределенных неуправляемых факторов. Вследствие влияния последних в момент производства прогноза неизвестны ни вероятности результатов прогноза (или известны с недостаточной точностью), ни, возможно, даже сами результаты. В первом случае речь идет о неопределенных факторах стохастической природы (т.е. о недостаточно изученных стохастических параметрах, относительно которых отсутствует необходимая статистическая информация), во втором - о неопределенных факторах не стохастической природы.

Прогнозы в условиях определенности и прогнозы в условиях неопределенности можно рассматривать как предельные случаи прогнозов в условиях риска, причем первые соответствуют очень малому риску, а вторые - очень большому.

Производство трех указанных видов прогнозов зависит от информации, имеющейся в распоряжении прогнозиста. В информационном аспекте согласно проведенной классификации все прогнозы можно разбить на прогнозы в условиях полной информации, прогнозы в условиях частичной информации и прогнозы в условиях отсутствия информации.

Чтобы выбрать оптимальный вариант из спектра альтернативных, нужен как можно больший объем информации. Качество прогноза зависит от полноты и адекватности имеющейся информации, и стремление повысить его приводит к необходимости получения новой или подтверждения имеющейся информации. Кроме того, информацию никогда нельзя получить без определенных затрат. Поэтому в каждой конкретной ситуации надо соразмерять ценность полученной дополнительной информации затратами на ее формирование. Если при производстве прогноза объем информации превышает требуемый или вновь подтверждается и без того достоверная информация, то улучшение качества прогноза вряд ли оправдает затраты на получение дополнительной информации.

Рассмотрим в общих чертах методы, применяемые при производстве прогнозов каждого типа. Прежде всего, отметим, что схема его практической реализации строго разработана лишь для детерминированного случая. Прогнозы в условиях риска и условиях неопределенности обычно сводятся к тому, что стохастичность или неопределенность тем или иным способом заменяются детерминированной схемой. Правомерность «детерминизации» задачи и адекватность детерминированной схемы реальной задачи в значительной мере обусловлены опытом и искусством прогнозиста. Для снижения степени субъективности в подобных случаях обычно применяются процедуры экспертных опросов.

При прогнозировании технико-экономических параметров в условиях определенности, когда прогнозирующая система обеспечена всей необходимой информацией протекания процесса, общая постановка задачи прогнозирования позволяет непосредственно применить весь богатый арсенал методов прогнозирования, и данный прогноз не вызывает возникновения концептуальных проблем, а его сложность зависит в основном от теоретических и вычислительных трудностей, носящих формальноматематический характер.

Более сложным видом прогноза является прогноз в условиях неполной определенности 
или в условиях риска. В этом случае прогнозируемый параметр подвержен влиянию факторов стохастической природы, относительно каждого из которых известен закон распределения, т.е. набор возможных значений фактора и вероятность их реализации. В указанных ситуациях широко используется прием сведения стохастической задачи к детерминированной. При этом возможны два способа детерминизации.

Первый способ - детерминизация на уровне факторов. Он состоит в том, что вероятностная картина приближенно заменяется детерминированной путем введения вместо стохастических факторов каких-то усредненных величин, носящих неслучайный характер. Как правило, в роли таких усредненных характеристик выступают математические ожидания стохастических факторов. Этот способ применяется при грубых, оценочных расчетах, а также в тех случаях, когда дисперсия наблюдаемых значений стохастических факторов сравнительно мала, т.е. они могут рассматриваться как неслучайные величины.

Если дисперсия значений стохастических факторов велика и замена каждого из них его математическим ожиданием может привести к большим ошибкам, применяется другой способ - детерминизация на уровне прогнозируемого параметра. Для этого на основании известного закона распределения значений стохастических факторов определяется закон распределения значений прогнозируемого параметра, после чего производится усреднение последнего. Очевидно, при линейной постановке задачи этот способ дает те же результаты, что и детерминизация на уровне факторов. Применение данного способа не исключает риска, т.е. возможного несовпадения реального значения параметра с прогнозом, причем оценкой меры риска может служить дисперсия значений параметра.

Таким образом, при решении задач стохастического прогнозирования возникают, во-первых, концептуальная проблема выбора способа детерминизации задачи, во-вторых, проблема формально-математического характера, связанная с выбором метода прогнозирования и его реализацией для детерминированной задачи.

Наиболее сложным видом прогноза является прогноз в условиях неопределенности. Он производится в ситуациях, когда на прогнозируемый параметр влияют (помимо детерминированных и стохастических) неопределенные факторы, информация относительно которых неизвестна в момент производства прогноза. При действии детерминированных факторов связь между ними и прогнозируемым параметром однозначна; каждой конкретной реализации детерминированного фактора соответствует единственное, вполне определенное значение параметра.

В случае же действия неопределенных факторов каждая их конкретная реализация приводит к тому, что прогнозируемый параметр принимает одно из нескольких возможных значений, заранее неизвестное. В этом отличие неопределенных факторов от детерминированных и некоторое их сходство со стохастическими.

Принципиальное различие между неопределенными стохастическими факторами заключается в том, что относительно последних прогнозист располагает полной статистической информацией, которой достаточно для определения вероятностей возможных исходов, поэтому стохастические факторы можно считать полностью изученными прогнозистом. Относительно же неопределенных факторов необходимая информация отсутствует, и в момент производства прогноза не известны ни вероятности результатов прогноза, ни, возможно, сами результаты.

Всю совокупность неопределенных факторов можно в свою очередь разбить на две группы: неопределенные факторы стохастической природы, для которых вероятности возможных результатов имеют физический смысл, но в момент производства прогноза либо неизвестны прогнозисту, либо известны с недостаточной степенью точности, и неопределенные факторы не стохастической природы, характеризующиеся тем, что в условиях их действия вероятности возможных результатов не имеют физического смысла. В первом случае сложность состоит в недостаточной изученности влияния этих факторов, во втором - в том, что задача прогнозирования не может быть поставлена и решена в терминах теории вероятностей.

Задачи прогнозирования и принятия решений в условиях неопределенности стали решаться лишь в последнее время, поэтому математический аппарат для их решения представляют сравнительно молодые направления исследования операций - теория игр, теория мини-макса, теория статистических решений.

Одной из основных задач прогнозирования 
динамики поведения технико-экономических параметров производства является предсказание их значений в зонах неопределенности, т.е. в таких областях их значений, в которых отсутствуют достоверные данные наблюдений. Наиболее часто такие задачи возникают при проведении реорганизации производства, при вовлечении в производство новых видов сырья или оборудования, при выпуске новых видов продукции при определении потребности в рабочей силе и других проблем.

Почти полное отсутствие информации в названных ситуациях объясняется либо полной невозможностью проведения соответствующих экспериментов на производстве для получения достоверной информации, либо тем, что подобные эксперименты слишком дорогостоящи и связаны, как правило, с остановкой основного производственного процесса на предприятии. Поэтому представляется целесообразным проведение математического анализа по оценке возможных решений с прогнозированием поведения технико-экономических показателей производства в условиях неопределенности. Такой анализ, как правило, позволяет сократить число проводимых экспериментов, подтверждающих или отрицающих полученные теоретические выводы.

Существующие методы прогнозирования $[1,4,7]$ поведения технико-экономических параметров в зонах неопределенности (или в зонах риска) не учитывают возможность неоднозначного представления достоверной информации в зонах определенности, где имеется большое число наблюдений. Учесть эту неоднозначность и выбрать наилучший из вариантов представления можно только путем использования принципов адаптации прогнозов [5,6].

Рассмотрим следующую ситуацию. Пусть задан некоторый объект или процесс, поведение которого определяют контролируемые параметры $\mathrm{X}_{1}, \ldots, \mathrm{X}_{\mathrm{n}}$, неконтролируемые параметры $\mathrm{Z}_{1}$, ..., $\mathrm{Z}_{\mathrm{p}}$; а поведение объекта описывают параметры $\mathrm{Y}_{1}, \ldots, \mathrm{y}_{\mathrm{k}}$.

Будем считать, что контролируемые параметры $\mathrm{X}_{\mathrm{i}}, \mathrm{i}=1, \ldots, \mathrm{n}$ могут варьироваться в двух непересекающихся областях: $A_{i}$ - область определенности параметра $\mathrm{X}_{\mathrm{i}}$; $\mathrm{B}_{\mathrm{i}}$ - область неопределенности параметра $\mathrm{X}_{\mathrm{i}}$ (где отсутствует достоверная информация). Необходимо получить адекватное представление поверхностей откликов $\mathrm{Y}_{\mathrm{j}}=\mathrm{F}_{\mathrm{j}}\left(\mathrm{X}_{1}, \ldots, \mathrm{X}_{\mathrm{n}}\right), \mathrm{j}=1,2, \ldots, \mathrm{k}$ и в случае, когда параметры $\mathrm{X}_{\mathrm{i}}$ варьируются в зонах неопределенности.

Процесс составления прогнозов с использованием принципов адаптации можно разбить на следующие основные этапы.

1. Предварительный этап, на котором описываются зоны $\mathrm{A}_{\mathrm{i}}$ и $\mathrm{B}_{\mathrm{i}}, \mathrm{i}=1, \ldots, \mathrm{n}$, выявляются законы распределения величин $\mathrm{X}_{\mathrm{i}}$ в зонах определенности, устанавливается статистическая независимость контролируемых параметров $\mathrm{X}_{\mathrm{i}}$ в зонах определенности.

2. Этап проведения дисперсионного анализа в зонах определенности. На этом этапе определяют для каждого $\mathrm{Y}_{\mathrm{j}}, \mathrm{j}=1, \ldots, \mathrm{k}$ влияют ли или нет выбранные факторы $\mathrm{X}_{\mathrm{i}}, \ldots, \mathrm{X}_{\mathrm{n}}$ на значение отклика, а также степень их влияния. Устанавливают, имеет или нет место эффект взаимодействия уровней различных факторов. Для количественных факторов, существенно влияющих на отклик $\mathrm{y}_{\mathrm{j}}$, выбирают минимально допустимые интервалы квантирования, при которых наблюдаются различия в откликах, при соседних уровнях этих факторов. С помощью соответствующим образом подобранных контрастов выбирают виды зависимостей между количественными факторами и откликом $\mathrm{y}_{\mathrm{j}}$. В случае наличия эффекта взаимодействия количественных факторов устанавливается форма связи взаимодействия этих факторов. С помощью аппарата дисперсионного анализа после составления дисперсионной таблицы можно получить ответы на следующие вопросы:

- влияют ли фиксированные уровни каждого фактора на прогнозируемые параметры и какова степень их влияния?

- каков минимально допустимый интервал квантирования количественных факторов в области, примыкающей к зоне неопределенности, при котором наблюдаются различия в откликах для соседних уровней этих факторов?

После применения описанных процедур мы имеем четко определенный и упорядоченный по степени влияния на прогнозируемые параметры перечень основных факторов, для каждого из которых известен интервал квантирования в области, примыкающей к зоне неопределенности.

3. Этап проведения регрессионного анализа [2,3]. На этом этапе с помощью метода наименьших квадратов устанавливается наилучшая форма связи между параметрами, характеризующими поведение объекта, и параметрами, 
определяющими его поведение, т.е. определяется вид функций

$$
\mathrm{y}_{\mathrm{j}}=\mathrm{F}_{\mathrm{j}}\left(\mathrm{X}_{1}, \ldots, \mathrm{X}_{\mathrm{n}}\right)
$$

и выдается оценка тесноты связи в области определенности параметров $\mathrm{X}_{\mathrm{i}}, \mathrm{i}=1, \ldots, \mathrm{n}$. При проведении регрессионного анализа используются результаты двух предыдущих этапов.

4. Этап прогнозирования искомых параметров. Осуществляется прогнозирование параметров $\mathrm{y}_{\mathrm{j}}, \mathrm{j}=1, \ldots, \mathrm{k}$ в области неопределенности путем экстраполяции на нее функций $\mathrm{F}_{\mathrm{j}}\left(\mathrm{X}_{1}, \ldots\right.$, $\mathrm{X}_{\mathrm{n}}$ ). Производится оценка качества прогноза путем сравнения расчетных результатов либо с имеющимися данными, либо с экспертными оценками. При наличии существенных расхож- дений между такими двумя наборами показателей осуществляется следующий этап.

5. Этап адаптации вида функций $\mathrm{F}_{\mathrm{j}}\left(\mathrm{X}_{1}, \ldots\right.$, $\mathrm{X}_{\mathrm{n}}$ ) к имеющимся данным из областей неопределенности либо к существующим экспертным оценкам. На этом этапе в качестве математического аппарата определения вида функций $\mathrm{F}_{\mathrm{j}}\left(\mathrm{X}_{1}, \ldots, \mathrm{X}_{\mathrm{n}}\right)$ выступает аппарат квадратичного программирования, который позволяет ранжировать результаты наблюдений, наложить ограничения на скорости изменения отклика в зависимости от изменения параметров $\mathrm{X}_{\mathrm{i}}, \mathrm{i}=1, \ldots, \mathrm{n}$, наиболее полно учесть существующую априорную информацию.

\section{Библиографический список}

1. Вайну Я.Я. Корреляция рядов динамики. М.: Статистика, 1977, 119с.

2. Дрейпер Н., Смит Г. Прикладной регрессионный анализ. Множественная регрессия. М.: Диалектика. 2007. 912c.

3. Песаран М., Слейтер Л. Динамическая регрессия. Теория и алгоритмы. М.: Финансы и статистика. 1984. 310c.

4. Статистическое моделирование - прогнозирование //под редакцией А.Г.Гранберга//. М.: Финансы и статистика, 1990. 490с.

5. Суменков М.С. Оптимальное планирование деятельности промышленных предприятий. М.: Издательство « Наука», 1980. 232с.

6. Суменков М.С., Суменков С. М. Прогнозирование динамики экономических параметров предприятия на основе нейронных сетей. М.: Журнал «Экономические науки», 2012, № 2. с. 90-94.

7. Форстер Э., Ренц Б. Методы корреляционного и регрессионного анализа. М.: Финансы и статистика, 1983. 302c. 